\title{
Pesquisa y control del cáncer cérvico-uterino en el Servicio de Salud de Valdivia (1993-2003)
}

\author{
Sergio G uzmán C, Paola Salas $\mathbf{R}^{\mathrm{a}}$, Raúl Puente $\mathbf{P}$, \\ Humberto Hott A, Eduardo Israel A, René G uzmán $S$. \\ Assessment of cervical cancer \\ screening in the Health Service of \\ Valdivia, Chile
}

Background: One of the best Public Health achievements in Chile has been the coverage of the program for detection and treatment or cervical cancer. Aim: To evaluate the results of the Cervical Cancer Screening Program in Valdivia Health Service (Southern Chile) between 1993 and 2003. Material and methods: Retrospective review of the number of women in whom a Papanicolau smear was obtained in the last three years. The incidence of the disease was calculated using pathological diagnoses of precursor or invasive lesions. Mortality for cervical cancer was calculated using data from death certificates. Results: During 2003, 79\% of women age 25 to 64 years, and 77\% of women aged 35 to 63 years (considered the highest risk group) were screened. We confirmed that the number of precursor lesions detected were increased along with the increasing screening coverage. The diagnosis of carcinoma in situ increased almost three times when compared with 1990 figures. Likewise, the diagnosis of early invasive cervical cancer behaved similarly. Mortality by cervical cancer also experienced a decrease, reaching 4.6 per 100.000 women over 15 years old in the Health Service of Valdivia. Conclusions: The sustained increase in cervical cancer screening coverage has had a favorable impact in the morbidity and mortality caused by the disease (Rev Méd Chile 2005; 133: 685-92).

(Key Words: Cervix neoplasms; Managed care programs; Mass screening)

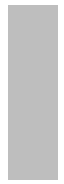

Recibido el 14 de septiembre, 2004. Aceptado en versión corregida el 30 de marzo, 2005. Servicio de Obstetricia y Ginecología, Hospital Clínico Regional de Valdivia e Instituto de Obstetricia y Ginecología, Facultad de Medicina de la Universidad Austral de Chile. Valdivia, Chile.

aMatrona, Magíster en Salud Pública.

$\mathrm{U}$ no de los logros más importantes de las políticas sanitarias de nuestro país, ha sido el desarrollo que ha alcanzado el programa de pesquisa y control del cáncer cervical-uterino, enfermedad cuya historia natural permite detectar

Correspondencia a: Dr. Sergio Guzmán C. Instituto de Ginecología y Obstetricia, Universidad Austral de Chile. Fono-Fax (63) 221693. E-mail: sguzman@uach.cl y reconocer precozmente sus lesiones precursoras $\mathrm{y}$, por lo tanto, acceder a tratamientos oportunos y eficaces, con especial impacto sobre la morbimortalidad de la mujer.

Actualmente, en el mundo, el cáncer cérvicouterino causa alrededor de 500.000 muertes anuales $^{1}$, situación que no es pareja, ya que los países de alto desarrollo sanitario han logrado disminuir, en forma muy significativa, su incidencia gracias a 
programas de pesquisa bien implementados ${ }^{2,3}$. Chile, a pesar de disponer de un programa cada vez más consolidado, aún muestra una situación desfavorable y la mortalidad por esta enfermedad, con una tasa de 9,6 por 100.000 mujeres, sólo está por debajo de la de México, Barbados y otros países del Caribe ${ }^{4,5}$.

En el Servicio de Salud Valdivia, el modelo que se está desarrollando desde hace 15 años, ha tenido como propósito a mediano y largo plazo, disminuir la incidencia de los estadios invasores de la enfermedad y reducir así la morbilidad y mortalidad por esta causa. Creemos que esto es posible en la medida en que el programa permita la detección y el tratamiento oportuno de sus lesiones precursoras.

El objetivo de este trabajo es dar a conocer los resultados del programa y el impacto que este ha tenido sobre las mujeres beneficiarias del sistema público de salud en la provincia de Valdivia durante los últimos 11 años.

\section{MATERIAL Y MÉTODO}

Hemos estudiado en forma retrospectiva los resultados del programa de pesquisa y control del cáncer cérvico-uterino del Servicio de Salud Valdivia, entre 1993 y 2003, es decir, durante 11 años. Con anterioridad, existía un programa que por defectos en su diseño fue considerado insatisfactorio y, por lo tanto, reemplazado por el actual en 1993.

El universo estuvo constituido por la población femenina mayor de 15 años, beneficiaria del Servicio de Salud, de acuerdo a la proyección anual del Instituto Nacional de Estadística (INE), cuyos datos pueden verse en la Tabla $4^{6}$. Esto corresponde a la población femenina expuesta a riesgo de esta enfermedad y que, para el año 2003, fue de 130.067 mujeres.
La pesquisa se realizó mediante el examen clínico y el estudio de la citología exfoliativa del cérvix obtenida en los consultorios de atención primaria del Servicio, mediante espátula de Ayre y teñida con el método de Papanicolaou (Pap) en el Laboratorio de Histología del Hospital Clínico Regional de Valdivia (HCRV).

Los casos clínica o citológicamente sospechosos fueron derivados a la Unidad de Patología Cervical (UPC) del HCRV, para efectuarse un estudio colposcópico y biópsico que permitiera llegar a un diagnóstico histológico.

Las variables estudiadas comesponden a la cobertura anual con Pap vigente, es decir, aquellas mujeres con un Pap realizado durante los últimos tres años, según normativa del Ministerio de Salud; las que fueron distribuidas en dos grupos etáreos: las de 2564 años durante los 11 años que se analizan y las de 35-64 años, consideradas como de mayor niesgo, registradas sólo desde el año 2000 en adelante.

Para el cálculo de la incidencia de la enfermedad, se consideraron sólo los diagnósticos histológicos de lesiones precursoras e invasoras, las que fueron clasificadas de acuerdo a normas internacionales y utilizadas para registrar incidencia y otros indicadores de proceso. En la Tabla 1, se resume la nomenclatura a que hacemos referencia. La tasa de incidencia se obtuvo del número de casos nuevos de la enfermedad, dividido por el total de mujeres expuestas cada año, multiplicado por 100 mil.

La mortalidad por cáncer cérvico uterino fue verificada mediante la revisión de los certificados de defunción del Registro del Servicio de Salud Valdivia y de las historias clínicas del HCRV y la tasa de mortalidad fue calculada con el número de muertes por esta causa, dividido por el total de la población expuesta cada año multiplicado por 100 mil.

Fueron incluidos los casos que histológicamente correspondían a cáncer cérvico-uterino, y

\section{Tabla 1. Pesquisa y diagnóstico de las lesiones neoplásicas del cuello uterino. N omenclatura internacional}

$\begin{array}{ll}\text { Pesquisa citológica: } & \text { Probable lesión de bajo grado. } \\ & \text { Probable lesión de alto grado. } \\ \text { Diagnóstico histológico } & \text { Neoplasia intraepitelial de bajo grado (NIE I) } \\ & \text { Neoplasia intraepitelial de alto grado (NIE II y III) } \\ & \text { Cáncer invasor }\end{array}$

The Bethesda System (TBS 2001).

Sociedad Internacional de Patología Ginecológica. 
sólo aquellos que tenían su domicilio en la provincia de Valdivia.

La recolección de la información fue hecha revisando los registros estadísticos sobre muestras citológicas de la atención primaria, del Servicio de Anatomía Patológica y de la Unidad de Patología Cervical del HCRV. Los resultados fueron analizados por los autores en reuniones periódicas.

\section{RESULTADOS}

El incremento global del número de Pap tomados en la última década, en el Servicio de Salud
Valdivia, ha alcanzado a $17 \%$, teniendo su expresión máxima durante 2003, en que se tomaron 26.127 muestras (Tabla 2).

En lo que respecta a la calidad de las muestras citológicas, la misma Tabla señala que hasta el año 2000, ellas fueron bastante satisfactorias, mostrando, sin embargo, un deterioro desde el año 2001 a 2003. Así lo evidencia el progresivo porcentaje anual de frotis menos que óptimo, es decir, de muestras citológicas sin componente endocervical, observadas durante los últimos tres años del programa.

En cuanto a cobertura, en la Figura 1 se muestra que ésta, medida a través del porcentaje de Pap

Tabla 2. Exámenes anuales de Pap y calidad de la muestra citológica, Servicio de Salud Valdivia

\begin{tabular}{|cccccc|}
\hline Año & $\begin{array}{c}\text { Total Pap } \\
\text { anuales } \\
n\end{array}$ & \multicolumn{2}{c}{ Frotis } & \multicolumn{2}{c|}{$\begin{array}{c}\text { Frotis menos } \\
\text { que óptimo** }\end{array}$} \\
& $\mathrm{n}$ & $\mathrm{n}$ & $\%$ & $\mathrm{n}$ & $\%$ \\
\hline 1993 & 22.296 & 129 & 0,5 & 519 & 2,1 \\
1994 & 23.168 & 154 & 0,6 & 542 & 2,3 \\
1995 & 158 (n)cuado* & 680 & 4,3 \\
1996 & 15.977 & 213 & 1,2 & 981 & 5,7 \\
1997 & 17.139 & 518 & 2,5 & 511 & 2,5 \\
1998 & 20.336 & 584 & 3,2 & 645 & 3,5 \\
1999 & 18.370 & 800 & 4,3 & 805 & 4,3 \\
2000 & 18.510 & 1.222 & 6,0 & 964 & 4,8 \\
2001 & 20.217 & 1.253 & 5,4 & 3.221 & 13,9 \\
2002 & 24.933 & 554 & 2,4 & 4.443 & 19,2 \\
2003 & 23.124 & 395 & 1,5 & 6.683 & 25,6 \\
\hline
\end{tabular}

Fuente: Cito-expert, Anatomía Patológica HCRV.

*Frotis inadecuado: no cumple con criterios para lectura citológica. ${ }^{* *}$ Frotis menos que óptimo: sin componente citológico endocervical.

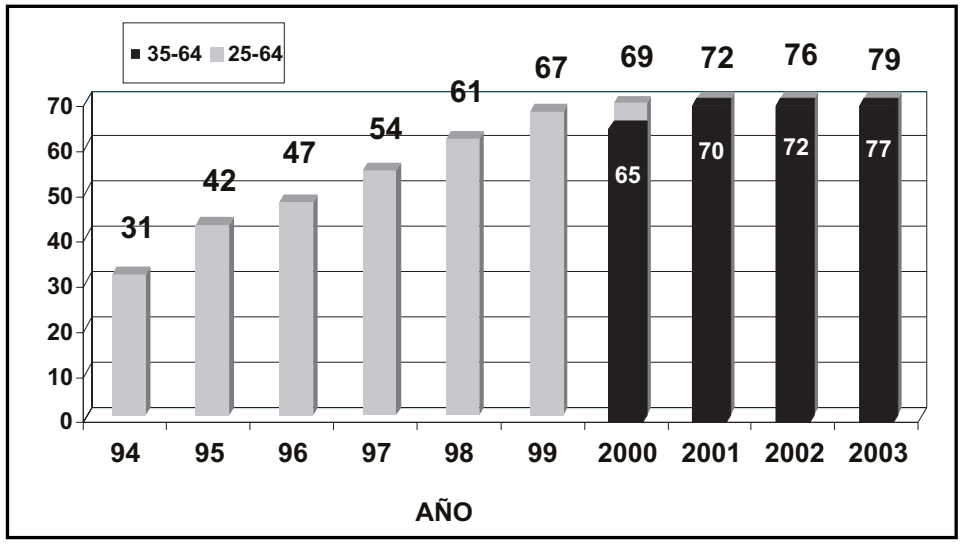

FIguRA 1. Pap vigente en mujeres de 25 a 64 años. Porcentaje anual. Servicio de Salud Valdivia, 1994-2003. 


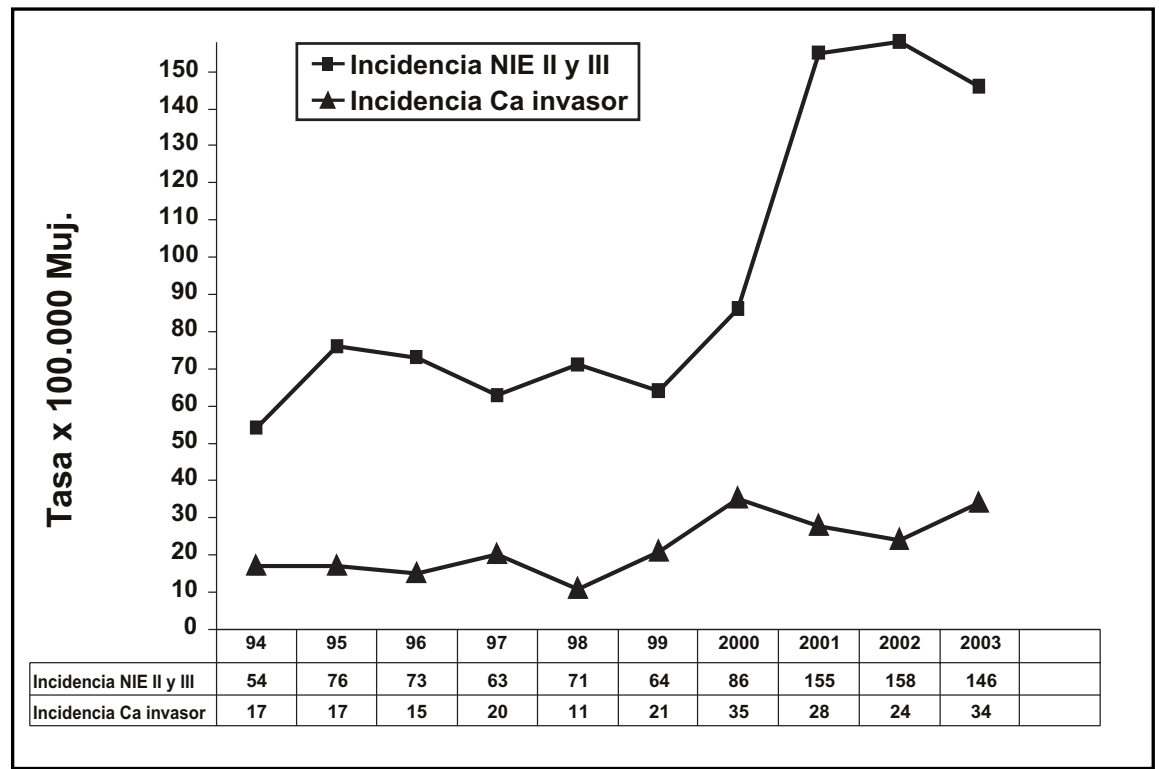

FiguRa 2. Incidencia de lesiones precursoras de alto grado y cáncer invasor. Tasa por 100.000 mujeres mayores de 15 años. Servicio de Salud Valdivia, 1994-2003.

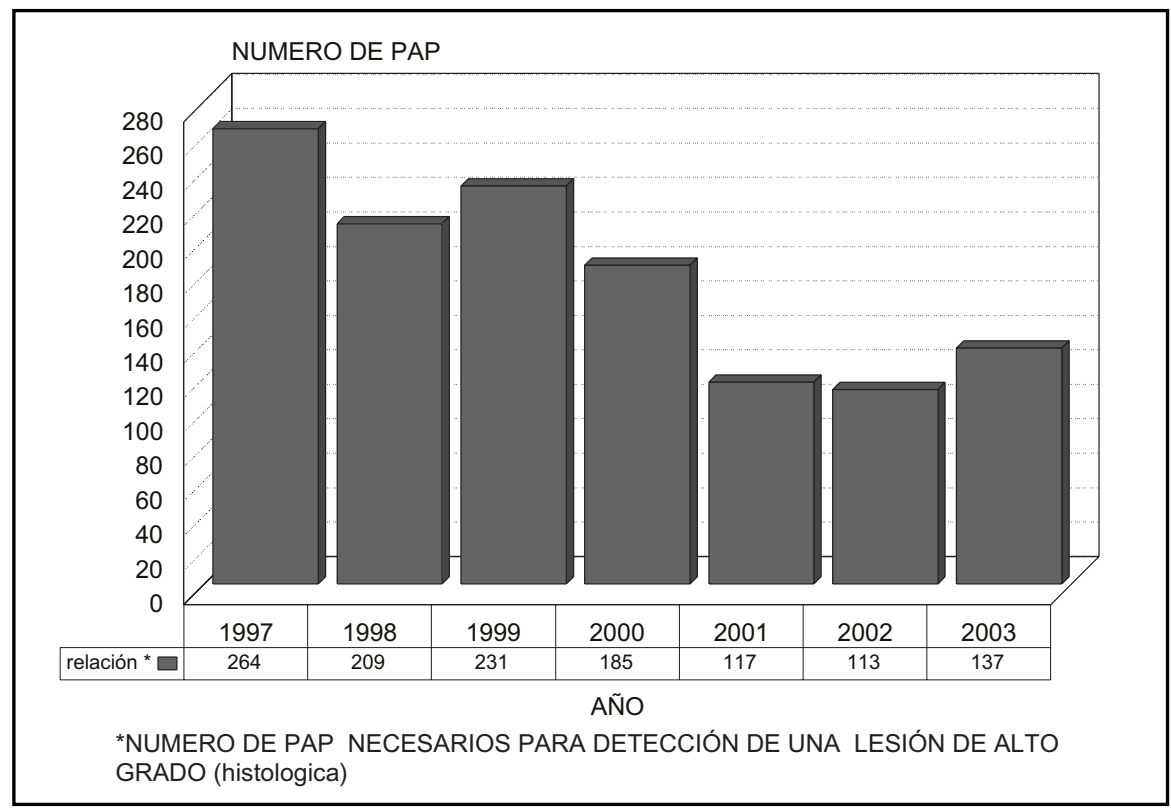

FIguRa 3. Número de Pap requeridos para diagnosticar una lesión de alto grado, Servicio de Salud Valdivia, 1997-2003. Base de datos computacional Cito-expert y biopsias Servicio de Anatomía Patológica Hospital Clínico Regional Valdivia. 
vigente en las mujeres de 25 a 64 años, ha tenido un aumento anual sostenido, significando un incremento de 48\% entre 1994 y 2003. En el grupo de mayor riesgo, cuyas edades están entre los 35-64 años, la cobertura (tabulada sólo desde 2000 en adelante), el incremento alcanzó a 12\% durante los últimos cuatro años de aplicación del programa.

Respecto a incidencia y en relación a cobertura, las Figuras 2 y 3 muestran que el diagnóstico de lesiones de alto grado aumenta en el tiempo, así como la eficacia de la pesquisa citológica. En
1997 se requería 264 exámenes citológicos para diagnosticar una lesión de alto grado; en 2003 se necesitaron 137 exámenes para el mismo objetivo (Figura 3).

La Tabla 3 muestra la incidencia de lesiones de alto grado (NIE II y III) y de cáncer invasor diagnosticadas anualmente y la proporción de ambos tipos de lesiones a través de los años que se evalúan en esta comunicación.

En la Figura 4 se observa la frecuencia anual de estadios de invasión precoz (I y II) y la de los

Tabla 3. N úmero y proporción de N IE III, lesiones de alto grado (LAG) y cáncer invasor diagnosticadas en el Servicio de Salud Valdivia, 1993-2003

\begin{tabular}{|cccccc|}
\hline Año & $\begin{array}{c}\text { NIE III } \\
\text { № }\end{array}$ & $\begin{array}{c}\text { Cáncer Invasor } \\
\text { № }\end{array}$ & $\begin{array}{c}\text { Proporción } \\
\text { NIE III/Ca invasor }\end{array}$ & $\begin{array}{c}\text { LAG } \\
\text { № }\end{array}$ & $\begin{array}{c}\text { Proporción } \\
\text { LAG/Ca invasor }\end{array}$ \\
\hline 1993 & 25 & 41 & 0,6 & 153 & 3,7 \\
1994 & 49 & 20 & 2,4 & 64 & 3,2 \\
1995 & 38 & 20 & 1,9 & 90 & 4,5 \\
1996 & 48 & 18 & 2,6 & 88 & 4,8 \\
1997 & 48 & 25 & 1,9 & 77 & 3,1 \\
1998 & 46 & 14 & 3,3 & 88 & 6,3 \\
1999 & 36 & 26 & 1,4 & 80 & 3,1 \\
2000 & 72 & 45 & 1,6 & 109 & 2,4 \\
2001 & 100 & 36 & 2,8 & 198 & 5,5 \\
2002 & 95 & 31 & 3,1 & 204 & 6,2 \\
2003 & 102 & 45 & 2,3 & 190 & 4,2 \\
\hline
\end{tabular}

Fuente: Diagnóstico Histológico, Anatomía Patológica, Hospital Clínico Regional Valdivia.

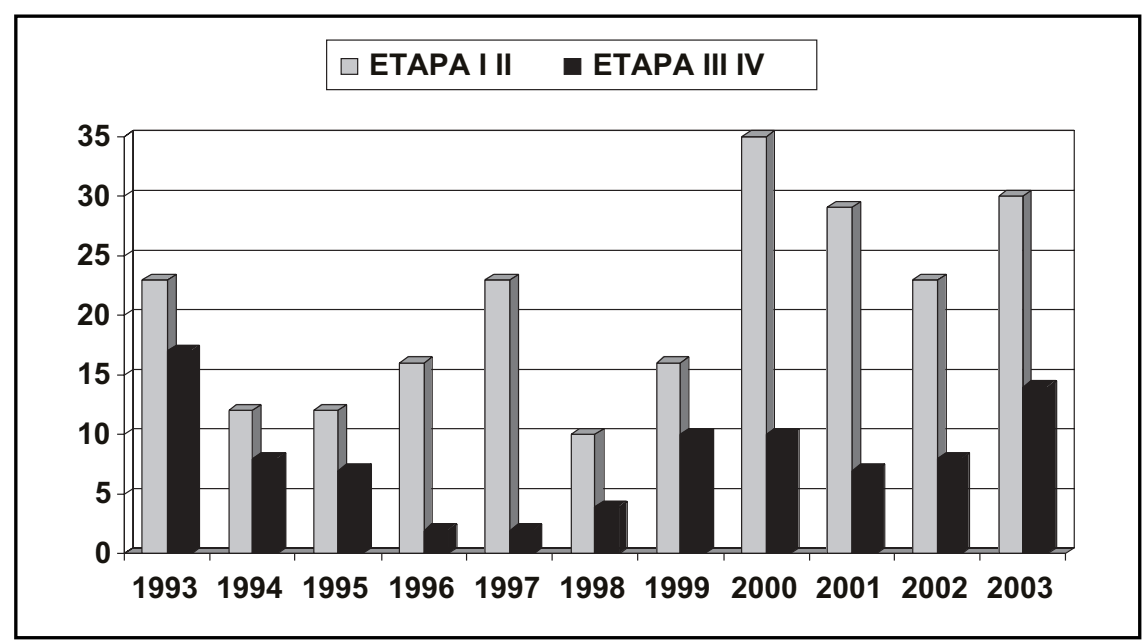

FiguRA 4. Cáncer cérvico uterino invasor. Etapas precoces (I y II) y etapas avanzadas (III y IV), Servicio de Salud Valdivia, 1993-2003. Diagnóstico Histológico, Servicio Anatomía Patológica, Hospital Clínico Regional Valdivia. 
estadios de invasión avanzada (III y IV) en tanto que la mortalidad, puede verse en la Tabla $4 \mathrm{y}$ Figura 5.

\section{DisCUSIÓN}

La reorientación estratégica, basada en criterios de eficiencia y eficacia recomendados por OMS/OPS, que ha sido aplicada gradualmente en el Programa de Pesquisa y Control del Cáncer de Cuello Uterino del Servicio de Salud Valdivia desde 1992 en adelante, ha sido, sin duda, el factor fundamental que ha permitido alcanzar una mayor cobertura de Pap vigente en la población femenina beneficiaria mayor de 15 años (17\%).

Esto se ha logrado mejorando la organización y la gestión en toda la provincia, con estrategias probadas en países desarrollados ${ }^{7-9}$, que contemplan acciones de promoción de la salud, talleres para la formación de monitores, educación continua a beneficiarias, visitas de reforzamiento a los
Tabla 4. M ortalidad por cáncer cérvico uterino, tasa por 100.000 mujeres mayores de 15 años. Servicio de Salud Valdivia 1993-2003

\begin{tabular}{|cccc|}
\hline Año & $\begin{array}{c}\text { Población } \\
\text { femenina } \\
\mathrm{n}\end{array}$ & Casos & $\begin{array}{c}\text { Tasa por } \\
\text { 100 mil } \\
\text { mujeres }\end{array}$ \\
\hline 1993 & 115.964 & 22 & 18,9 \\
1994 & 117.350 & 23 & 19,5 \\
1995 & 118.692 & 15 & 12,6 \\
1996 & 120.089 & 14 & 11,6 \\
1997 & 121.455 & 19 & 15,6 \\
1998 & 122.792 & 20 & 16,2 \\
1999 & 124.096 & 16 & 12,8 \\
2000 & 125.405 & 18 & 14,3 \\
2001 & 126.983 & 11 & 8,6 \\
2002 & 128.532 & 10 & 7,8 \\
2003 & 130.067 & 6 & 4,6 \\
\hline
\end{tabular}

Fuente: Certificados de defunción. Servicio de Salud Valdivia.

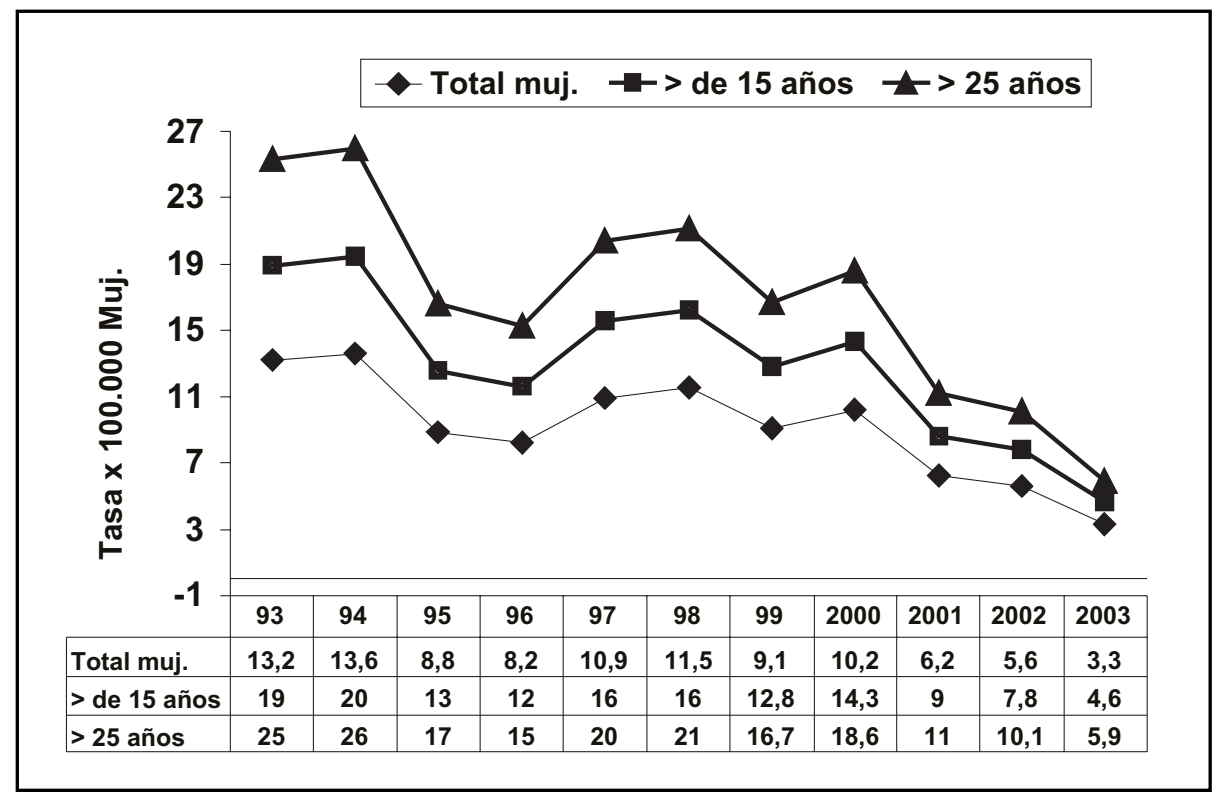

FIgURA 5. Mortalidad por cáncer cérvico-uterino. Tasa total por 100.000 mujeres y tasas específicas por edad. Servicio de Salud Valdivia, 1993-2003. Certificados de defunción, Servicio de Salud Valdivia. 
consultorios de atención primaria, toma de Pap en estaciones de salud rural, difusión del programa por canales de comunicación, salidas a la comunidad a través de un Pap móvil, retroalimentación expedita mediante la instalación de bases de datos computacionales en los establecimientos de atención primaria y secundaria, y talleres anuales de evaluación conjunta en el Servicio de Salud.

Ha sido este conjunto de medidas lo que ha permitido alcanzar en Valdivia la cobertura de $79 \%$ en las mujeres entre 25 y 64 años y de $77 \%$ en el grupo de mayor riesgo, conformado por mujeres entre 35 y 64 años (Figura 1).

Esta mejoría substancial en la cobertura constituye un acierto sanitario, ya que ha permitido diagnosticar anualmente un mayor número de lesiones intraepiteliales de alto grado (NIE II y III), a pesar que aún no se observa una disminución de la incidencia de cáncer invasor (Tabla 3).

Sin embargo, es satisfactorio ver que, respecto a cáncer invasor, cada año se han diagnosticado más mujeres con estadios invasores precoces (I y II) que con estadios invasores avanzados (III y IV), lo que epidemiológicamente es esperable en un programa que está teniendo éxito. Esto permite esperar, si el programa mejora, que las mujeres con etapas III y IV desaparezcan en el corto plazo, con el consiguiente beneficio para nuestra población femenina.

La observación de un aumento del porcentaje de frotis informados como menos que óptimo, es

\section{REFERENCIAS}

1. Franco EL, Franco ED, Ferenczy A. Cervical cancer: epidemiology, prevention and the role of human papillomavirus infection. CMAJ 2001; 164: 1017-25.

2. Quinn M, BabB P, Jones J, AuEn E. Effect of screening on incidence of and mortality from cervical cancer in England: evaluation based on routinely collected statistic. BMJ 1999; 318: 904.

3. MacGregor JE, Campbell MK, Mann EM, Swanson KY. Screening for cervical intraepithelial neoplasia north east Scotland shows fall in incidence and mortality from invasive cancer with concomitant rise in preinvasive disease. BMJ 1994; 308: 1407-11. decir, muestras citológicas sin componente endocervical durante los últimos 3 años, constituye preocupación, y aún no tenemos una clara explicación al respecto. No obstante, como esto significa un desmedro de la eficiencia, ya que requiere de la repetición del Pap en el corto plazo, es materia que debe analizarse más detenidamente ${ }^{10}$.

El aumento anual de los exámenes citológicos en una enfermedad cuya historia natural implica una progresión gradual desde lesiones intraepiteliales de alto grado (NIE II y NIE III) a invasoras, hace necesario alcanzar y mantener, al menos, una cobertura global de $80 \%$ de Pap vigente entre la población de riesgo ${ }^{11,12}$. Esto no es fácil, pero con las estrategias que se están llevando a cabo, creemos estar muy cerca de obtenerla.

El progresivo descenso de la tasa de mortalidad por cáncer cérvico uterino entre la población femenina mayor de 15 años, expuesta al riesgo, desde 18,9 por 100 mil en 1993, a 4,6 por 100 mil en 2003, es otro hecho estimulante, porque representa un logro del trabajo en equipo y nos demuestra que es posible modificar favorablemente nuestros indicadores sanitarios, a pesar de la habitual insuficiencia de recursos.

No hemos logrado aún las tasas de mortalidad que muestran Canadá, Estados Unidos de Norteamérica, Islandia, Finlandia y Suecia, pero si alcanzáramos una cobertura sobre $80 \%$ como ya fue planteado, mejoraremos sustancialmente ${ }^{13}$.

4. Serra I, Serra L, Castro R, García V, Serra J, Decinti E ET AL. Cáncer cérvico-uterino, un desafío sin resolver. Rev Chil Obstet Ginecol 1997; 62: 75-85.

5. Suárez E, Prieto M, Rojas II, Fernández B, Prado R, Olfos P. Programa nacional de cáncer cérvico uterino. Rev Chil Obstet Ginecol 2001; 66: 480-91.

6. Instituto Nacional de Estadísticas, Ministerio de Economía Chile: Estimaciones y proyecciones de población por sexo y edad. Total país: 19502050.

7. DaY NE. Screening for cancer of the cervix. J Epidemiol Comm Health 1989; 43: 103-6.

8. Parkin DM. Cancer in developing countries. Cancer Surv 1994; 19-20: 519-61.

9. Womack SD, Chirenje ZM, Blumenthal PD, Gaffikin L, Mcgrath Ja, Chipato T et al. Evaluation of a 
human papillomavirus assay in cervical screening in Zimbabwe. BJOG 2000; 107: 33-8.

10. Capurro I, Rojo J, Pino T, Vásquez C, Garay J, Venegas M. Programa de detección y control de cáncer de cuello uterino en Servicio de Salud Araucanía Sur. Rev Chil Obst Ginecol 2002; 67: 114-20.

11. Richart RM. Natural history of cervical intraepithelial neoplasia. Obstet Gynecol 1990; 75: 131-3c.
12. Gorey KM, Holowaty EJ, Fehringer G, Laukkanen E, Moskowitz A, WeBster DJ, Richter NL. An international comparison of survival: Toronto, Ontario and Detroit, Michigan, metropolitan areas. Am J Public Health 1997; 87: 1156-63.

13. SASIENI P, Cuzick J, Farmery E. Accelerated decline in cervical cancer mortality in England and Wales. Lancet 1995; 346: 1566-7.

\section{Agradecimientos}

A las matronas que laboran en la atención primaria y que constituyen el pilar fundamental en el logro de los objetivos de detección precoz del programa de cáncer cérvico uterino, a través de la toma del Papanicolaou y el compromiso en otorgar salud a las mujeres de sus comunidades. 